\title{
MicroRNA-145 inhibits tumorigenesis and invasion of cervical cancer stem cells
}

\author{
XI ZHOU ${ }^{1,2}$, YAN YUE $^{2}$, RENXIAO WANG ${ }^{2}$, BAOLAN GONG ${ }^{2}$ and ZHAO DUAN ${ }^{1}$ \\ ${ }^{1}$ Department of Obstetrics and Gynecology, The Second Affiliated Hospital of Xi'an Jiaotong University, \\ Xi'an, Shaanxi 710004; ${ }^{2}$ Department of Obstetrics and Gynecology, Renmin Hospital, \\ Hubei University of Medicine, Shiyan, Hubei 442000, P.R. China
}

Received November 14, 2016; Accepted January 2, 2017

DOI: $10.3892 /$ ijo.2017.3857

\begin{abstract}
MicroRNA (miR)-145 has been reported to induce cancer stem cell (CSC) differentiation through downregulation of the stem cell transcription factors (TFs) that maintain CSC pluripotency. High expression of miR-145 indicates a good prognosis in cancer patients, but its role in cervical cancer stem cells (CCSCs) is not known. We show that expression of miR-145 and core stem cell transcription factors, Sox 2, Nanog and Oct4, are associated with the pluripotency of CCSCs, with increased expression of miR-145 after cervical tumorsphere (CT) differentiation. miR-145 overexpression inhibited expression of core TFs, as well as decreasing tumor invasion and colony formation, whereas miR-145 knockdown led to the opposite effects. Injection of adenovirus-miR-145 significantly reduced tumor growth in nude mice. High miR-145 expression predicted a better prognosis compared with that in patients with low miR-145 expression after analyses of The Cancer Genome Atlas (TCGA) data. These results suggest that miR-145 is able to induce CT differentiation through enzymolyzing TFs and might be a therapeutic target for cervical carcinoma.
\end{abstract}

\section{Introduction}

Cervical cancer (CC) is the most common cancer in females and the second most common cause of cancer-related mortality in China (1). Cervical cancer is the fourth most common cancer in females in 'developed' countries (2). The poor prognosis of cervical cancer is associated with its highly invasive and diffuse metastatic characteristics $(3,4)$. The cervical cancer stem cell (CSCC) is recognized as being the 'seed' for cancer metastasis (5). However, the CSCC was not identified until

Correspondence to: Professor Zhao Duan, Department of Obstetrics and Gynecology, The Second Affiliated Hospital of Xi'an Jiaotong University, Xi'an, Shaanxi 710004, P.R. China

E-mail:zduan09@sina.com

Key words: microRNA-145, Sox2, Nanog, Oct4, cervical cancer stem cell recently because of a shortage of special cell-surface markers. High expression of stem cell transcription factors (TFs) in cancer is recognized as a cancer stem cell (CSC) marker and correlates with a poor prognosis (6). Core stem cell TFs such as Foxd3 (7), Sox9 (8), Sox2 (9,10), Nanog (11) and Oct4 (10) have been reported to be highly expressed in cervical cancer, and to be associated with increased invasion of cancer cells and carcinogenesis. It has been assumed that downregulation of expression of these TFs by certain molecules (e.g., miRNAs) can inhibit invasion and metastasis by cancer cells (12).

Previously, miR-145 was reported to induce differentiation of human embryonic stem cells through enzymolysis of the core stem cell transcription factors Oct4, Sox2, and KLF4 (13). It has also been proposed as a tumor-suppressor, and its expression is decreased in many types of cancer $(14,15)$, for example, in colonic adenocarcinomas where miR-145 expression is lower than in the mucosa (16). Intravesicular administration of exogenous miR-145 has been shown to inhibit growth of mouse orthotopic human bladder cancer xenografts (17), while miR-145 targets MUC13 to suppress the growth and invasion of pancreatic cancer cells (14). Overexpression of miR-145 promotes differentiation by inhibiting Oct4 expression in human endometrial adenocarcinoma cells (18). Based on these data, we investigated the expression and function of miR-145 in cervical cancer using a cervical tumorsphere (CT) model.

\section{Materials and methods}

Culture of $C T$. This study protocol was approved by the Medical Ethics Committees of Xi'an Jiaotong University of Medicine (no. H34-32-1, Xi'an, China). All investigations were conducted in accordance with the Declaration of Helsinki. All the patients involving this study provided a written informed consent. The nude mice used in this study were treated in accordance with the institutional guidelines of the Animal Ethics Committee for the care and use of animals in Xi'an Jiaotong University of Medicine, China. We confirm that all methods were carried out in accordance with relevant guidelines and regulations of Xi'an Jiaotong University of Medicine.

We previously described the method of CT culture (16). Briefly, 21 samples of cervical cancer tissue (stage IB, $n=14$; stage IC, $n=4$; stage IIa, n=3; age, 36-69 years) were obtained by resection (Table I). For CT culture, tumors were washed 
immediately in phosphate-buffered saline (PBS) containing $500 \mathrm{U} / 1$ penicillin G (Gibco; Thermo Fisher Scientific, Waltham, MA, USA) and $500 \mathrm{mg} / 1$ streptomycin (Gibco) $<30 \mathrm{~min}$ after resection, and then digested overnight in DMEM/F12 medium supplemented with $0.5 \mathrm{mg} / \mathrm{ml}$ collagenase IV (Gibco). Tumors were cultured in stem cell medium (DMEM/F12), $10 \mathrm{ng} / \mathrm{ml}$ basic fibroblast growth factor (bFGF), $10 \mathrm{U} / \mathrm{ml}$ leukemia inhibitory factor, $1 \times 10^{5} \mathrm{U} / 1$ penicillin, $100 \mathrm{mg} / \mathrm{l}$ streptomycin (all reagents from EMD Millipore, Merck KGaA, Darmstadt, Germany) at $37^{\circ} \mathrm{C}$ in a humidified atmosphere containing $5 \% \mathrm{CO}_{2}$. Clones of $>50$ cells were recognized as tumorspheres. These were dissociated every 7-10 days by incubation in a non-enzymatic cell dissociation solution (Sigma-Aldrich, St. Louis, MO, USA) for $2 \mathrm{~min}$ at $37^{\circ} \mathrm{C}$ and passaged at $1 \times 10^{3}$ cells per $100-\mathrm{mm}$ plate. Tumorsphere cells had differentiated completely by 8 days after switching to stem cell medium without bFGF.

Transduction of adenovirus vectors. All Ad-vectors had comparable titers of $10^{8}-10^{9}$ transducing units $/ \mathrm{ml}$. Virus suspensions were stored at $-80^{\circ} \mathrm{C}$ until use. Suspensions were centrifuged briefly and kept on ice immediately before use. For transduction, $2 \times 10^{4}$ dissociated tumorsphere cells were transduced 1 day after initial seeding of cells with a multiplicity of infection (MOI) of 25. Cells were incubated in stem cell medium containing adenovirus particles and $4 \mu \mathrm{g} / \mathrm{ml}$ polybrene (Santa Cruz Biotechnology, Inc., Santa Cruz, CA, USA) for $18 \mathrm{~h}$ at $37^{\circ} \mathrm{C}$ in a humidified atmosphere containing $5 \% \mathrm{CO}_{2}$. Ad-particles were removed and the medium replaced with fresh stem cell medium.

Colony formation assay. A colony formation assay was carried out as previously described (19). Briefly, single cervical carcinoma cells or dissociated tumorsphere cells were cultured in DMEM/F-12 medium supplemented with $10 \%$ fetal calf serum (FCS; Life Technologies, Carlsbad, CA, USA), 2 mmol/1 glutamine (Invitrogen, Carlsbad, CA, USA), $1 \times 10^{5} \mathrm{U} / 1$ penicillin, and $100 \mathrm{mg} / \mathrm{l}$ streptomycin. Cells were cultured at clonal densities of $100-300 / \mathrm{cm}^{2}$ on $2 \%$ gelatin (Sigma-Aldrich)-coated tissue culture dishes (BD Biosciences, San Jose, CA, USA) at $37^{\circ} \mathrm{C}$ in $5 \% \mathrm{CO}_{2}$ in air. Cloning plates were monitored every day and medium was changed every 2-3 days. After 28 days of culture, plates were fixed in $10 \%$ formaldehyde/PBS for $10 \mathrm{~min}$ and stained with Harris hematoxylin. Clones ( $>50$ cells) were counted on $\geq 3$ plates per sample and averaged. Efficiency of colony formation was determined as [(number of colonies)/(number of cells seeded)] x100.

Flow cytometric analyses. Dissociated tumorsphere cells or adenovirus vector-transfected cells were grown in 6-well plates and collected. Cells were resuspended in PBS containing $2 \%$ fetal bovine serum (FBS) and $0.1 \%$ sodium azide, and then analyzed by a FACScalibur system (BD Biosciences). Acquisition was set for 10,000 events per sample. Data were analyzed using FACS v4.1.2 (BD Biosciences). Triplicate samples were analyzed in each experiment.

Real-time polymerase chain reaction (PCR). Total RNA was extracted from cells using TRIzol ${ }^{\circledR}$ (Invitrogen). miRNA levels were assayed using Taqman ${ }^{\circledR}$ probes and primer sets
Table I. Patient characteristics.

\begin{tabular}{lcc}
\hline Sample no. & Age (years) & Diagnosis \\
\hline 1 & 36 & SCC, Stage IB \\
2 & 41 & SCC, Stage IB \\
3 & 49 & SCC, Stage IB \\
4 & 47 & SCC, Stage IB \\
5 & 56 & SCC, Stage IB \\
6 & 54 & SCC, Stage IB \\
7 & 57 & SCC, Stage IB \\
8 & 68 & SCC, Stage IB \\
9 & 56 & SCC, Stage IB \\
10 & 53 & SCC, Stage IB \\
11 & 36 & SCC, Stage IB \\
12 & 39 & SCC, Stage IB \\
13 & 49 & SCC, Stage IB \\
14 & 52 & SCC, Stage IB \\
15 & 61 & SCC, Stage IC \\
16 & 46 & SCC, Stage IC \\
17 & 67 & SCC, Stage IC \\
18 & 38 & SCC, Stage IC \\
19 & 66 & SCC, Stage IIA \\
20 & 63 & SCC, Stage IIA \\
21 & & SCC, Stage IIA \\
\hline & &
\end{tabular}

SCC, squamous cell carcinoma.

Table II. PCR primers and products.

\begin{tabular}{llc}
\hline Symbol & \multicolumn{1}{c}{ Primer } & $\begin{array}{c}\text { Product } \\
\text { (bp) }\end{array}$ \\
\hline Nanog & S: 5'-AATGGTGTGACGCAGAAG-3' & 255 \\
& A: 5'-AGATTCCTCTCCACAGTTATAG-3' & \\
Oct4 & S: 5'-AGCTGGAGAAGGAGAAGC-3' & 194 \\
& A: 5'-AAAGCGGCAGATGGTCGT-3' & \\
Sox2 & S: 5'-CAATAGCATGGCGAGCGG-3' & 196 \\
& A: 5'-GTCGTAGCGGTGCATGGG-3' & \\
GAPDH & S: 5'-AAGGCTGAGAATGGGAAAC-3' & 254 \\
& A: 5'-TTCAGGGACTTGTCATACTTC-3' & \\
\hline
\end{tabular}

S, sense; A, antisense.

(Applied Biosystems, Foster City, CA, USA) according to the manufacturer's instructions. Briefly, first-strand cDNA was generated using a reverse transcription system kit (Promega, Madison, WI, USA) with random primers of miR-145. Realtime PCR was carried out using power SYBR Green PCR master mix (Applied Biosystems) in a StepOnePlus ${ }^{\circledR}$ system (Applied Biosystems). The level of glyceraldehyde 3-phosphate dehydrogenase (GAPDH) mRNA was used as the control for internal normalization. For exact quantification of gene copies 
Table III. Primers for overexpression of miR-145 or knocked down miR-145.

\begin{tabular}{lll}
\hline Symbol & Primer & \multicolumn{1}{c}{ Sequence } \\
\hline Pri-miR-145 & Sense & 5'-TGCTGGTCCAGTTTTCCCAGGAATCCCTGTTTTG \\
& & GCCACTGACTGACAGGGATTCGGGAAAACTGGAC-3' \\
& Antisense & 5'-CCTGGTCCAGTTTTCCCGAATCCCTGTCAGTCAGTGGCCA \\
& & AAACAGGGATTCCTGGGAAAACTGGACC-3' \\
Si-miR-145 & Abm & (Catalog number mh5195) \\
Si-miR-145 control & Abm & (Catalog number m010) \\
\hline
\end{tabular}

Table IV. Primers for mutation miR-145 target sequence on Sox2, Nanog and Oct4.

\begin{tabular}{ll}
\hline Symbol & \multicolumn{1}{c}{ Primers } \\
\hline Oct4 & S: GGAGTCGGGGTGGAGAGCAACTCC \\
& A: CCTCAACGAGAGGTGGGGCTGAGG \\
Nanog & S: CTTTAGTTAATTCATACAATGTC \\
& A: GACATTGTATGAATTAACTAAAG \\
Sox2 & S: TCTCCCCCCTCGCTGTCCGGCCCT \\
& A: AGGGCCGGACAGCGAGGGGGGAGA \\
\hline
\end{tabular}

$\mathrm{S}$, sense; A, antisense.

per cell, reverse-transcribed miR-145 cDNA was used as a template to formulate standard curves. Then, the exact number of copies of miR-145 per cell was calculated according to their molecular weight and cell counts. Primer sequences are presented in Table II.

Implantation of tumorspheres and tumorsphere-derived cells into nude mice. After dissociation of tumorspheres and tumorsphere-derived differentiated cells from 21 cervical cancer patients in a non-enzymatic cell-dissociation solution, cells were washed in serum-free Hank's balanced salt solution (HBSS). Then, cells were suspended in a 1:1 (v/v) mixture of serum-free DMEM/F12, and $1 \times 10^{5}$ cells were injected (s.c.) into the right (differentiated cells) or left (tumorsphere cells) mid-abdominal area of nude mice using a 23-G needle. Animals were subjected to necropsy 28 days after implantation, and tumor growth assessed by measuring volume using the formula: $\mathrm{V}=1 / 2 \times\left(\mathrm{L} \times \mathrm{W}^{2}\right)$. In the silenced-miR-145 group, mice were sacrificed at 14 days after injection to avoid necrosis in transplanted tumors. Sequences of primary miR-145 or silent miR-145 are shown in Table III.

Western blotting. Lysates were extracted from tumorspheres or cells. Proteins $(20 \mu \mathrm{g})$ were separated by $10 \%$ sodium dodecyl sulfate-polyacrylamide gel electrophoresis (SDS-PAGE) and transferred onto nitrocellulose membranes. The latter were blocked in 5\% skimmed milk in Tris-buffered salineTween-20, and then incubated with primary antibodies to core TF proteins or GAPDH (1:500 dilution; Santa Cruz
Biotechnology, Inc.) overnight at $4^{\circ} \mathrm{C}$. After incubation with horseradish peroxidase-labeled secondary antibodies, membranes were developed using a SuperSignal ${ }^{\circledR}$ West Pico Trial kit (Pierce, Rockford, IL, USA).

Immunohistochemical analyses. Cervical cancer samples were fixed in phosphate-buffered $10 \%$ formalin $(\mathrm{pH} \mathrm{7.2)}$, embedded in paraffin, and cut into sections $(4 \mu \mathrm{m})$. Sections were dewaxed in xylene, dehydrated in alcohol, and incubated in $0.01 \mathrm{M}$ sodium citrate buffer $(\mathrm{pH} \mathrm{6.0)}$ ) for antigen retrieval. Sections were incubated with $3 \% \mathrm{H}_{2} \mathrm{O}_{2}$ for 30 min to block endogenous peroxidase activity and with normal mouse serum at $37^{\circ} \mathrm{C}$ for 15 min to block non-specific binding of antibody. Then, sections were incubated with Sox 2, Oct4 or Nanog antibodies (1:100 dilution in PBS; Santa Cruz Biotechnology, Inc.) for $2 \mathrm{~h}$ at room temperature, followed by incubation with biotinylated secondary antibody (Santa Cruz Biotechnology, Inc.) and 3,3'-diaminobenzidine.

Mutation of miR-145 binding site within the sequence of Sox2, Nanog and Oct4. The complementary DNAs of Sox2, Nanog and Oct4 were purchased from Shanghai Yingji Biological Company (Shanghai, China). Mutated constructs containing the $6 \mathrm{bp}$ point mutations in the core TF seed sequence predicted to be the miR-145 target sequence were synthesized using a QuikChange II site-directed mutagenesis kit (Stratagene, La Jolla, CA, USA). The primers were designed by Stratagene using their own software (http://labtools.stratagene.com/QC) and are shown in Table IV. Ad-vectors expressing the core TF wild-type or mutation of miR-145 seed sequence under the control of the U6 promoter were produced by transient transfection of HEK293T cells, and then transfected into tumorspheres following the procedures described above.

Injection of Ad-miR-145 into exograft tumors in null mice. Titers of virus stocks were checked using TCID $_{50}$ methods. High-titer stocks were stored at $-70^{\circ} \mathrm{C}$ until use. Two weeks after injection of tumorspheres into nude mice, the tumors became palpable and each tumor site was injected with $5.8 \times 10^{5}$ pfu of Ad-miR-145 and Ad-Mock with gadolinium (1:1) (Sigma-Aldrich). Tumor size was measured each week.

Cell invasion assay. Cell invasion was evaluated using 24-well Transwell ${ }^{\circledR}$ culture chambers, as previously described (18). Cells were seeded at $5 \times 10^{4}$ per well and cultured in stem cell medium for $24 \mathrm{~h}$. Then, cells were fixed in methanol 
A

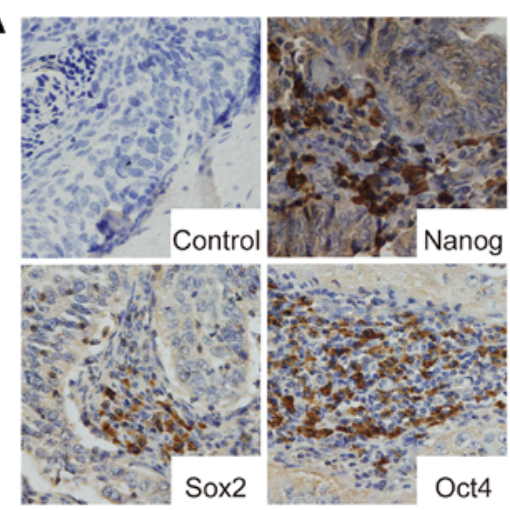

D

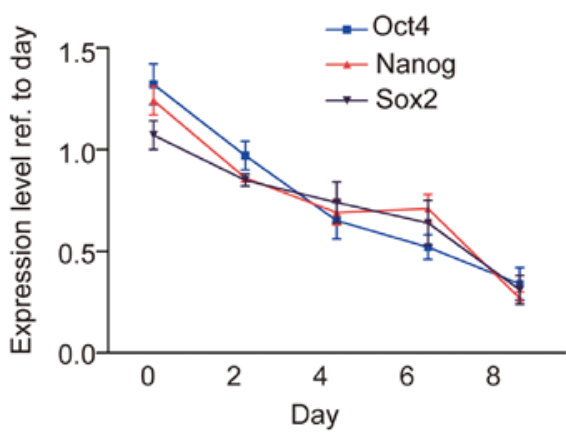

B

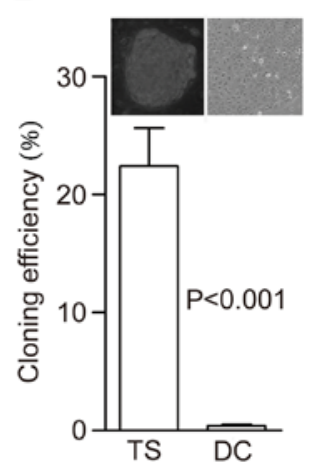

C

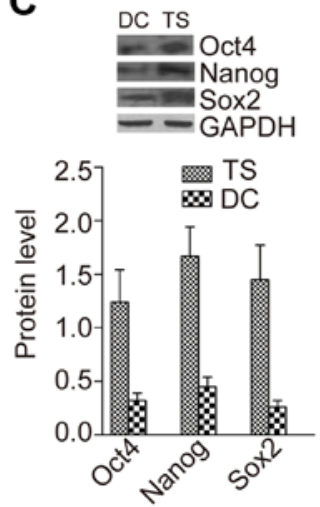

E

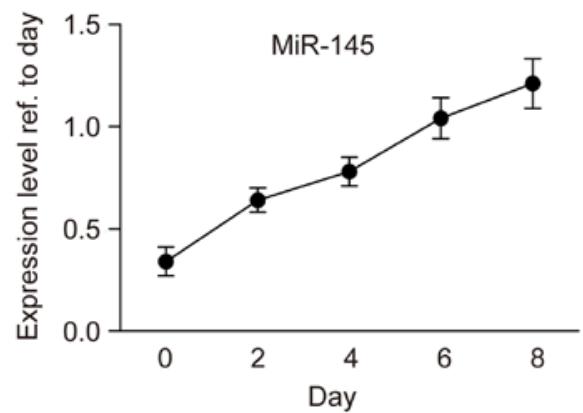

$\mathbf{F}$

miR-145 target sequence

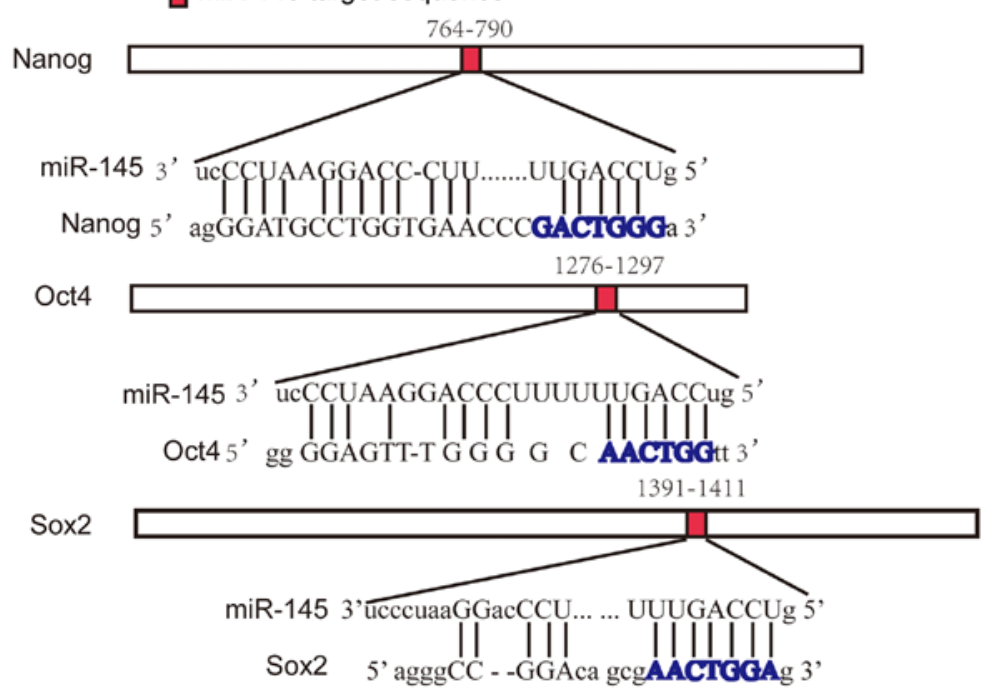

Figure 1. miR-145 expression is increased after differentiation of cervical tumorspheres. (A) In situ expression of core stem-cell transcription factors (TFs: Sox 2, Nanog, Oct4) in cervical carcinoma samples (x400 magnification). (B) Colony formation of cells dissociated from tumorspheres (TS) or differentiated tumorspheres (DC), $\mathrm{P}<0.001, \mathrm{n}=3$. (C) Expression of Sox2, Nanog and Oct4 in cells dissociated from tumorspheres (TS) or differentiated tumorspheres (DC). GAPDH was used as the loading control. (D and E) Relative expression of core TFs decreased and miR-145 level increased during CT differentiation, $\mathrm{n}=3$. (F) Prediction of miR-145 binding sequences on Oct4, Nanog, and Sox 2 transcripts by miRanda software.

and stained with $5 \%$ crystal violet. After examination under a light microscope, cells were eluted with $33 \%$ acetic acid. Optical-density values of the eluate were read using a Bio-Rad microplate reader at $590 \mathrm{~nm}$.

Patient database bioinformatics. The protocol followed that previously described (20). Briefly, data on expression of genes and miRNA were obtained from the Cancer Genome Atlas (TCGA; https://tcga-data.nci.nih.gov/tcga/tcgaHome2.jsp) for patients diagnosed with squamous cervical cancer. Expression of genes or miR-145 was divided into 'high' and 'low' groups based on the mean \pm 1 SD. Kaplan-Meier survival curves were generated comparing these two groups via the log-rank test. miR-145 expression combined with expression data for Oct4, Nanog, or Sox 2 were subtracted from the miR-145 data for each patient, and the analysis described above was repeated.

Statistical analyses. Data are shown as the mean \pm standard error of the mean. Comparison between groups were performed using analysis of variance, Fisher's exact test or 
A

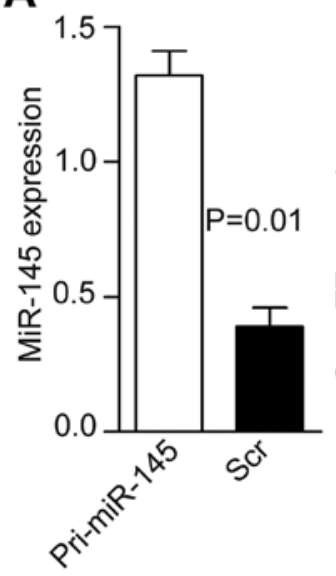

D

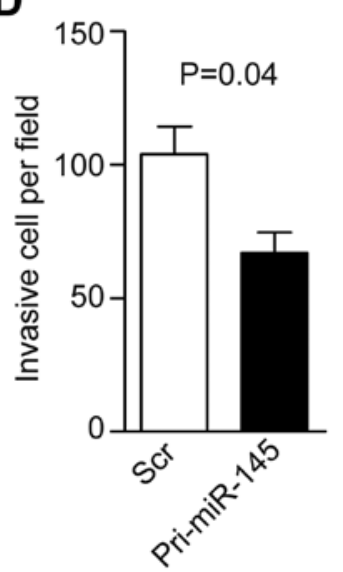

B

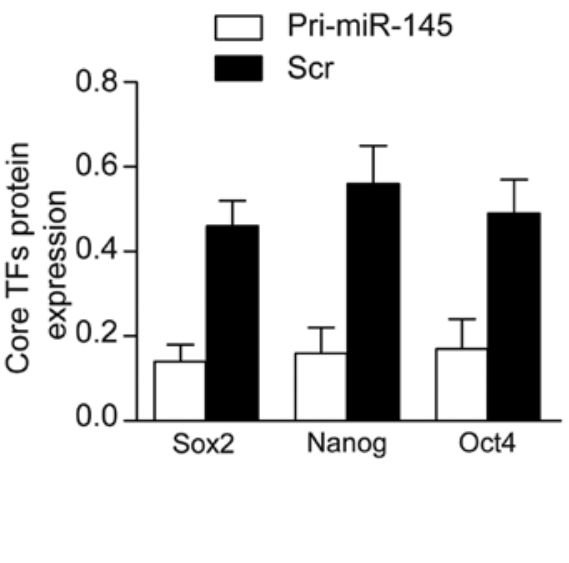

C

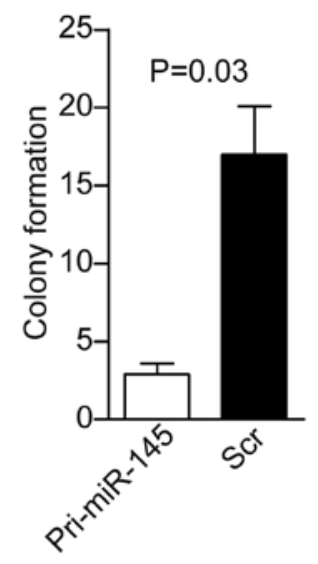

E

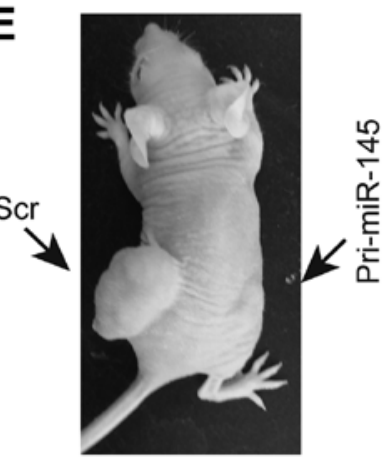

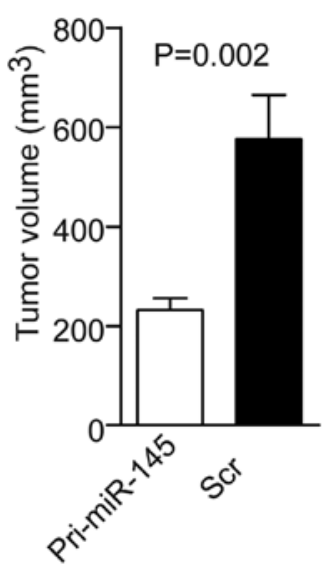

Figure 2. Overexpression of miR-145 induces CT differentiation and inhibits CT invasion and tumor formation. miR-145 level increased (A), while core TF levels (B) and colony formation (C) tumorsphere invasion (D) decreased after miR- 145 overexpression. $\mathrm{P}<0.05$ before and after transfection with Ad-pri-miR-145 (pri-miR-145), n=3. (E) Tumor formation of CTs harboring AD-pri-miR-145 or its control in nude mice, $P=0.02, n=5$.

two-tailed Student's t-test. $\mathrm{P}<0.05$ was considered to indicate a statistically significant difference.

\section{Results}

Expression of miR-145 and core stem cell TFs in undifferentiated cervical cancer stem cells. Our results showed that cervical cancer tissues were immunopositive for Nanog (20/21), Oct4 (19/21) and Sox2 (20/21), and 19/21 samples were positive for all three TFs. Staining for these TFs was visible in the cytoplasm and nuclei of a few (but not all) cancer cells, and we could detect that most cervical cancer cells expressed these TFs (18/21) (Fig. 1A). After enzymatic dissociation and culture in stem cell medium, a few colonies (tumorspheres) formed in all tissues tested (21/21) after 15 days. Colonies were formed at the rate of $22.41 \pm 3.23$ per 100 cells dissociated from tumorspheres, but this rate was reduced to only $0.41 \pm 0.07$ per 100 cells dissociated from differentiated tumorspheres $(\mathrm{P}<0.001)$ (Fig. 1B) when they detached from the disks. Furthermore, protein levels of Nanog, Sox 2 and Oct4 were significantly higher in tumorsphere cells than in differentiated cells $(\mathrm{P}=0.012$, Fig. $1 \mathrm{C})$.

Next, we examined expression of miR-145 and core TFs according to tumorsphere stages. First, we assessed expression of miR-145 in cells cultured under tumorsphere conditions or differentiated conditions [removal of basic fibroblast growth factor (bFGF) from the stem cell medium]. Quantitative reverse transcription-polymerase chain reaction (qRT-PCR) showed that the protein levels of core TFs decreased, whereas miR-145 level increased, at different time points and underwent dynamic changes (Fig. 1D and E). Two studies previously reported potential miR-145 binding sites in the genes of these TFs $(19,21)$, so we used miRanda software to predict miR-145 binding sites in the mRNAs of these TFs, the results confirmed potential miR-145 binding sequences within them (Fig. 1F).

Overexpression of miR-145 induces tumorsphere differentiation by decreasing expression of core TFs. Next, we investigated the role of miR-145 in cervical cancer. Ad-miR-145-GFP was transduced into tumorspheres or their differentiated cells to increase miR-145 expression. Flow cytometric analyses showed that there were $91 \pm 1.9 \%$ GFP-positive cells in the overexpressed Ad-miR-145-GFP group and $91 \pm 2.3 \%$ in the adenovirus-scrambled sequence-GFP (Ad-scr) group with no homology to the human genome, which acted as the control group among transfected tumorsphere cells. The miR-145 level was increased 4.5-fold in the overexpressed Ad-miR-145-GFP 


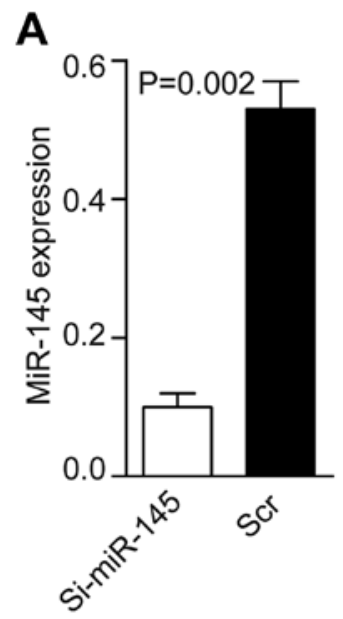

B $\quad$ C
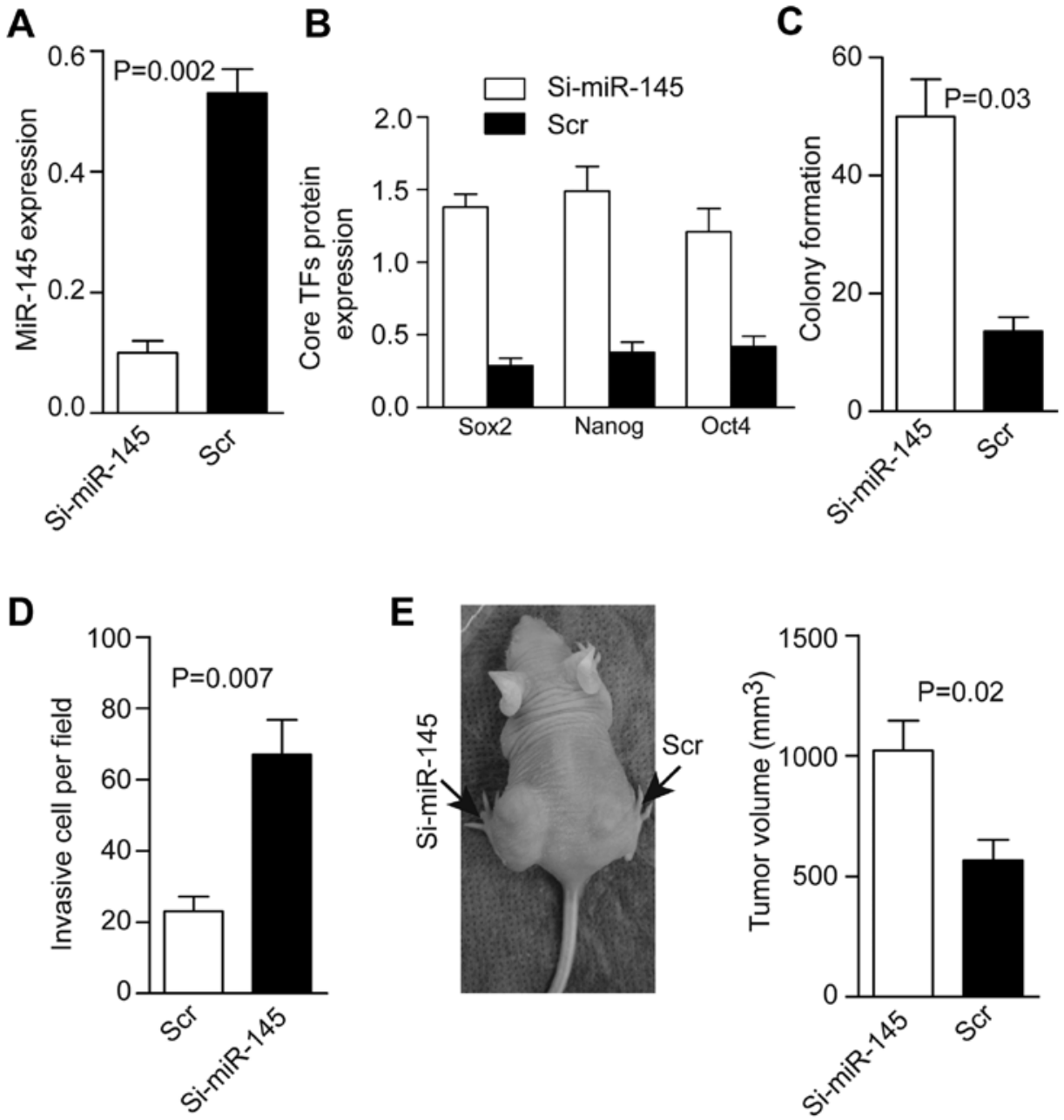

Figure 3. Knockdown of miR-145 maintains CT pluripotency and increases CT invasion and tumor formation. miR-145 level decreased (A), while core TF levels (B), colony formation (C) and tumorspheres (D) increased after knockdown of miR-145. P<0.05 before and after transfection with Ad-silence-miR-145 (si-miR-145), n=3. (E) Tumor formation increased after knockdown of miR-145. $\mathrm{P}<0.03$ before and after transfection with Ad-Silence-miR-145, $\mathrm{n}=5$.

group compared with its control (Fig. 2A). Accordingly, levels of core TFs were decreased significantly according to western blotting, and colony formation and cell invasion was decreased in the overexpressing Ad-miR-145-GFP group (Fig. 2B-D). Next, dissociated tumorsphere cells harboring Ad-miR145-GFP or Ad-scr-GFP were injected into nude mice. A total of 21 Ad-scr-GFP-tumorspheres derived from the 21 patients caused tumor formation in mice after 28 days. In contrast, 21 Ad-miR-145-GFP-tumorspheres from these 21 patients only caused 11 tumors, and those tumors were relatively small compared with those in the Ad-scr-GFP group (Fig. 2E).

Knockdown of miR-145 promotes tumorsphere tumorigenicity by maintaining $T F$ expression. Next, we transfected the Ad-silence-miR-145 (Ad-si-miR-145-GFP) vector or its control (Ad-scr-GFP) into CTs. Flow cytometric analyses showed that there were $90 \pm 1.4 \%$ GFP-positive cells in the Ad-si-miR145 -GFP group and $88 \pm 3.2 \%$ in the Ad-scr-GFP group. The miR-145 level was decreased fourfold compared with the control (Fig. 3A), whereas levels of TFs, colony formation, and cell invasion increased after silencing of miR-145 (Fig. 3B-D). Tumor formation was 21/21 in the Ad-si-miR-145 group and 20/21 in the scr-GFP group. To prevent tumor growth leading to necrosis (which would have affected the measurement of tumor volume), mice were sacrificed 14 days after injection. Tumors were larger in the si-miR-145 group than in the scr-GFP group (Fig. 3E).

Mutation of miR-145 binding sites within the sequences of Sox2, Nanog and Oct4 inhibit their miR-145-induced enzymolysis. miR-145 seed sequences were predicted within Oct4, Sox 2 3'-untranslated regions (UTR) and Nanog coding sequence (CDS) by the software miRanda after mutation of miR-145 target sequences ( $6 \mathrm{bp}$ deletion of the miR-145 target site) in these core transcription factors (Fig. 4A), and the vectors for the mutated structures are shown in Fig. 4B. First, we transfected Ad-Sox2-EGFP, Ad-Sox2-EGFP-mutation (mut)-miR-145 target sequence (Ad-Sox2-EGFP-mut) into tumorspheres, a luciferase reporter with no UTR of Sox2 and Oct4, CDS of Nanog were used as a negative control. Flow cytometric analyses revealed the percentage of EGFPpositive cells to be $86-89 \%$ ( $87 \pm 2.2 \%), 88-89 \%$ ( $88 \pm 0.6 \%)$, and $87-89 \%(88 \pm 1.9 \%)$, respectively. The Sox 2 level did not significantly differ between the wild-type (WT) group and the control (con) group by 24 or $48 \mathrm{~h}$ after transfection $(\mathrm{P}=0.47$ and 0.64, respectively), but Sox 2 expression in the WT group 
A

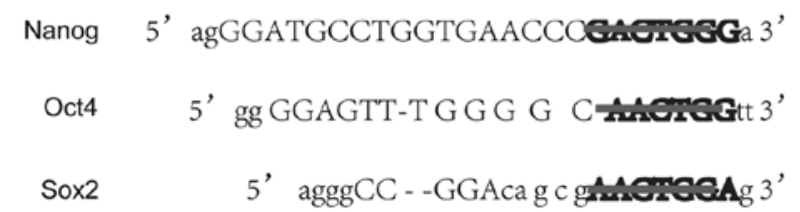

C Sox2

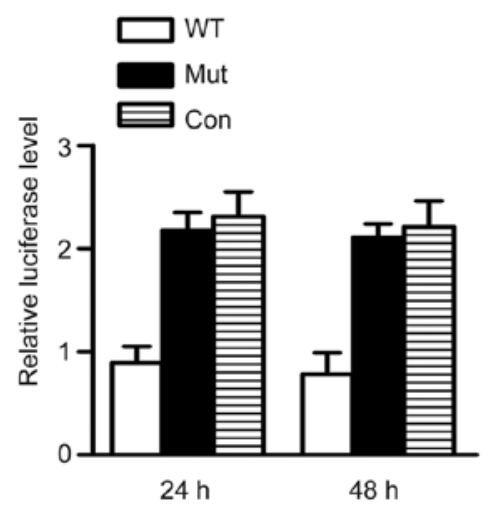

$\mathbf{F}$

Sox2

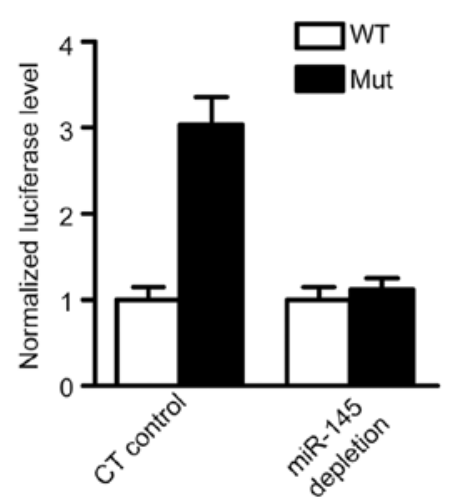

B

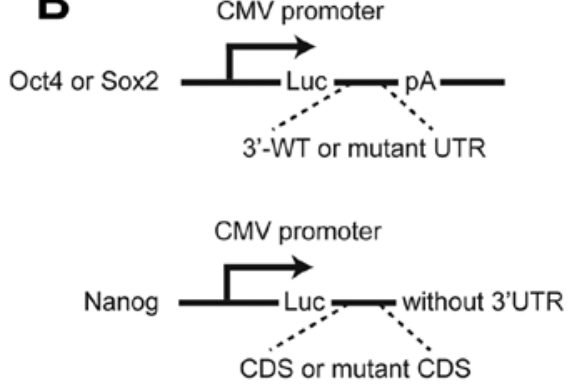

E
Nanog

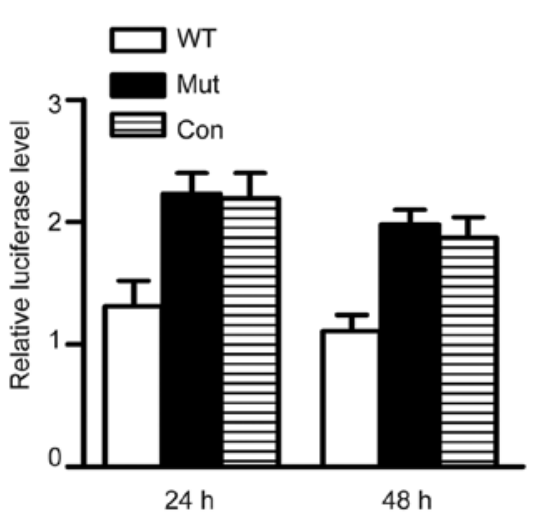

G

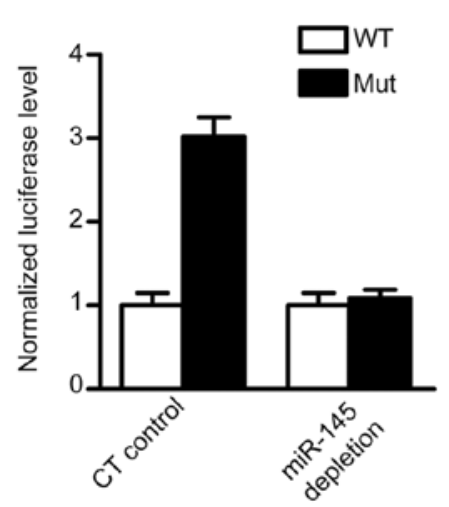

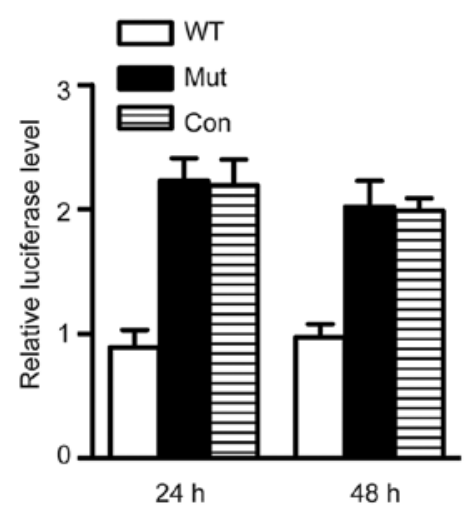

H

Hanog

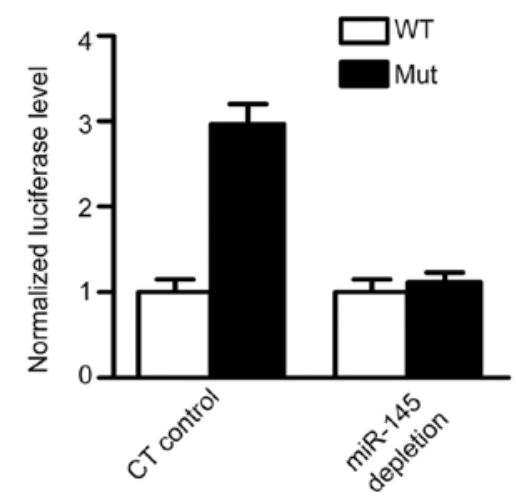

Figure 4. Endogenous miR-145 represses the 3'-untranslated regions (UTRs) of Oct4, Sox2, and coding sequence (CDS) of Nanog in CTs. (A) The mutational sequences in the core TFs. (B) The 3'-UTR or CDS reporters in target validation. Luc, firefly luciferase; pA, polyadenylation signal; WT, wild-type. Mutation (mut) has a 6 bp deletion of the miR-145 target site. The relative luciferase level of 3'-UTR luciferase reporters of Sox2 (C), Oct4 (D), and CDS luciferase reports of Nanog (E) in CTs under self-renewal conditions at 24 or $48 \mathrm{~h}$ after transfection. Mut, mutant UTR or CDS with a 6 bp deletion of the miR-145 target sites; Control, the basal luciferase reporter without the UTR of Sox 2 and Oct4, CDS of Nanog $(n=3)$. The difference between 3'-UTR mutant and wild-type luciferase reporters of Oct4 (F), Sox $2(\mathrm{G})$, and CDS mutant and wild-type luciferase reporters of nanog (H) depends on miR-145 in CTs. On the y-axis, the mutant reporter level is normalized against the average of the wild-type reporter level to reflect the magnitude of repression.

was lower than in the other two groups $(\mathrm{P}=0.012$ and 0.011 , respectively) (Fig. 4C). Similarly, following transfection of Ad-Oct4-EGFP, Ad-Oct4-EGFP-mut and Ad-control-EGFP, or Ad-Nanog-EGFP, Ad-Nanog-EGFP-mut and Ad-controlEGFP, flow cytometric analyses revealed the percentage of GFP-positive cells to be $83-86 \%(84 \pm 1.9 \%)$ and $83-87 \%$ $(85 \pm 1.7 \%)$, respectively. The Oct4 and Nanog levels did not differ between the mut group and the control group ( $\mathrm{P}=0.75$ and 0.57 , respectively), and their expression was lower in the WT groups than in the other groups $(\mathrm{P}=0.002$ and 0.005 , respectively) (Fig. 4D and E).
We further addressed the dependence of the reported repression of Oct4, Sox 2 UTR reporter, and Nanog CDS reporter on the level of miR-145. After depletion of miR-145 by antisense inhibitor, locked nuclei acid (LNA) in CT completely abolished the differential regulation between the mutant and WT 3'-UTR reporters in Oct4 and Sox 2 (Fig. 4F-H), and the mutant and wild-type CDS in Nanog. These results were in accordance with the reports by $\mathrm{Xu}$ et al (13) and Wang et al (21).

Ad-miR-145 inhibits the growth of tumors derived from cervical tumorspheres. Tumor cells were dissociated from 
A
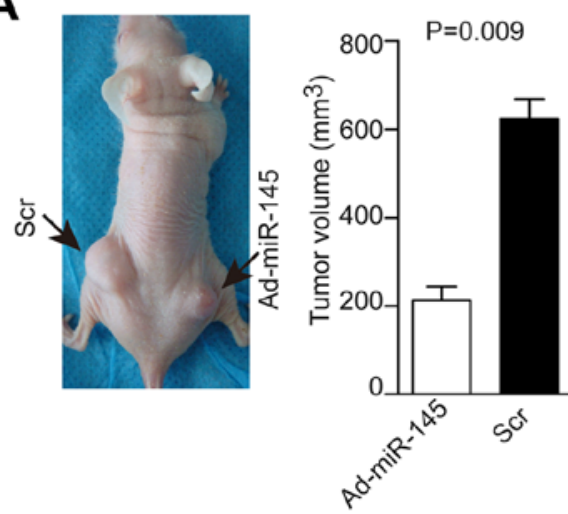

B

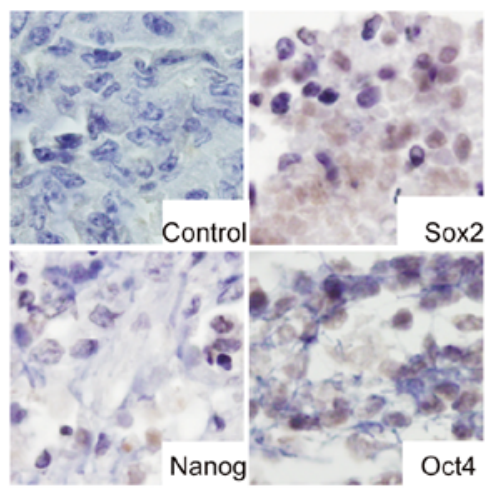

E

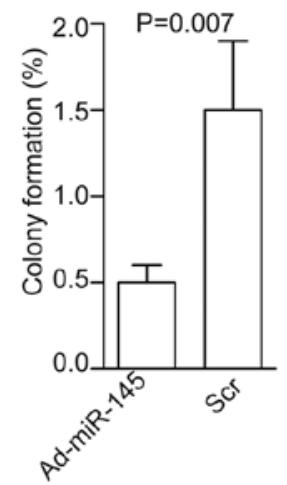

$\mathbf{F}$

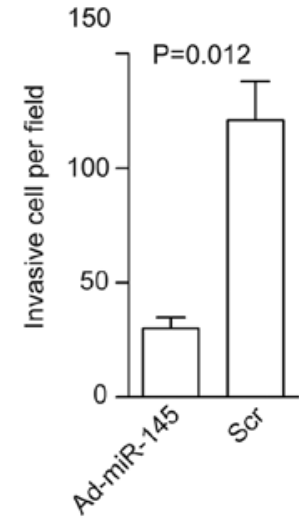

Figure 5. Injection of AD-miR-145 into transplanted tumors inhibits tumor growth, expression of core TFs, colony formation, and tumorsphere invasion. Injection of AD-miR-145 $\left(5.8 \times 10^{5} \mathrm{pfu}\right)$ into transplanted tumors inhibited tumor growth $(\mathrm{A}, \mathrm{P}=0.09)$, suppressed the expression of core TFs as shown by immunohistochemistry (B), decreased levels of core TFs $(C, P=0.02)$, increased miR-145 level $(D, P=0.04)$, and decreased colony formation $(E$, $P=0.007)$ and cell invasion $(\mathrm{F}, \mathrm{P}=0.012)$.

tumorspheres and injected into null mice. The resulting tumors were visible or palpable 2 weeks after injection. Next, we injected $5.8 \times 10^{5}$ pfu of Ad-miR145 or Ad-Mock with gadolinium combination into tumors three times a week (22). Mice were sacrificed at day 28 and tumor volume measured. Tumors were smaller in the Ad-miR-145 group than in the AD-scr group ( $\mathrm{P}=0.009$, Fig. 5A), the weak expression of core stem cell TFs was detected after Ad-pri-miR-145 treatment (Fig. 5B). Moreover, levels of core TFs were decreased $(\mathrm{P}=0.03$, Fig. 5C), miR-145 level was increased $(\mathrm{P}=0.04$, Fig. 5D), colony formation was decreased ( $\mathrm{P}=0.007$, Fig. 5E), and cell invasion was decreased $(\mathrm{P}=0.012$, Fig. $5 \mathrm{~F})$ in the Ad-miR-145 group compared with the control group.

High expression of miR-145 is associated with increased patient survival. To ascertain the clinical relevance of miR-145 and these core stem cell transcripts in the prognosis of CC patients, we evaluated the TCGA dataset. We found that high expression of miR-145 indicated a better prognosis compared with low expression of miR-145 (Fig. 6A). When miR-145 levels were combined with Nanog expression from the same patient, patients in the low Nanog/high miR-145 expression group had a better prognosis compared with the high Nanog/low miR-145 expression group (Fig. 6B). Similar results were obtained for expression of low miR-145 with high Sox 2 and Oct 4 in these subtypes compared with their opposite groups. These results implied that these genes could be informative for survival of CC patients (Fig. 6C and D).

\section{Discussion}

Cervical cancer is caused by human papillomavirus infection (HPV) (23). Overexpression of miR-145 in HPV-positive cervical cancer cells results in reduced genome amplification and late expression of genes (24). Herein, we focused on the relationship between miR-145 and mRNAs of core stem cell TFs in cervical cancer. The core stem cell TFs, Sox2, Nanog and Oct4, are essential for CSC maintenance. Downregulation of expression of these TFs induced CSC differentiation and reduced CSC tumorigenesis (25).

Wang et al reported downregulation of expression of miR-145 in cervical carcinoma, and suggested miR-145 to be involved in cervical carcinogenesis (26); this speculation was confirmed in our study. Moreover, we have provided the potential mechanisms by which miR-145 downregulates expression of core stem cell TFs, induces CCSC differentiation, and inhibits CCSC tumorigenesis. First, we examined expression of miR-145 and core stem cell TFs that maintain the pluripotency of CTs. miR-145 expression was increased significantly in differentiated cells compared with tumorspheres. In addition, we found a negative correlation between miR-145 levels and core TFs. These results strongly suggest 
A

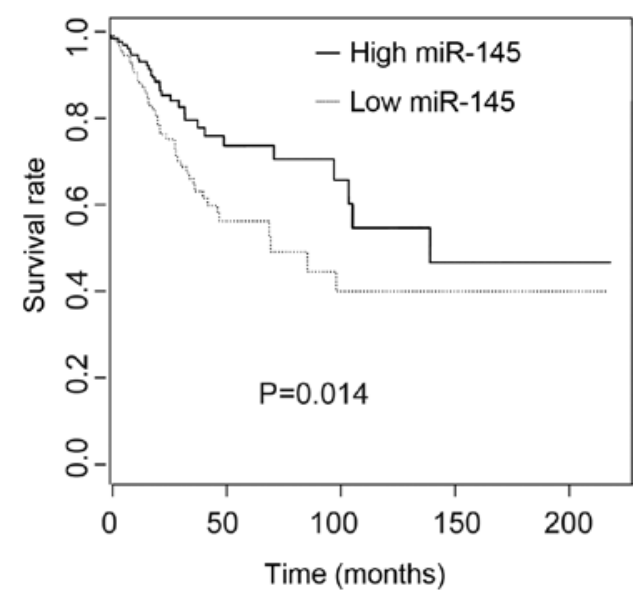

C

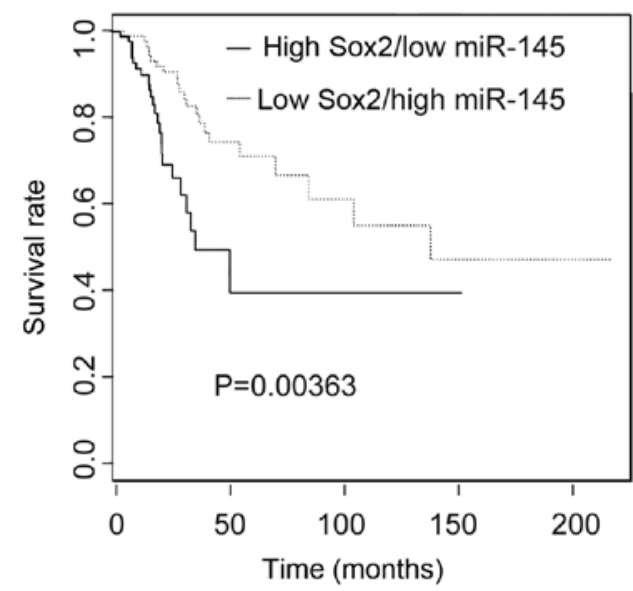

B

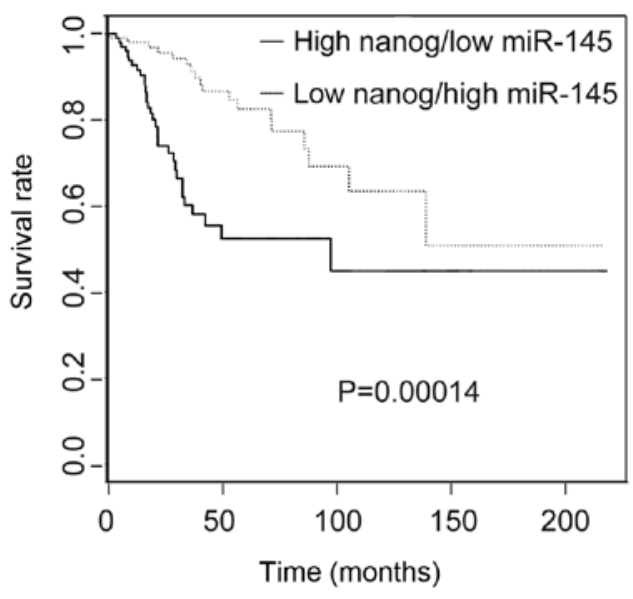

D

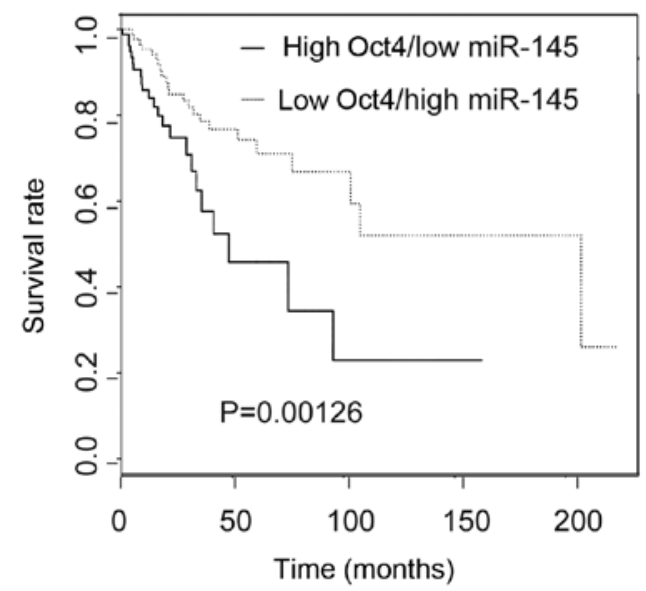

Figure 6. Survival curves were analyzed based on expression of miR-145 or core TFs in all SCC patients in the Cancer Genome Atlas (TCGA) dataset. (A) High levels of miR-145 suggests longer patient survival. A robust difference is seen when miR-145 is combined with the core stem cell transcription factors Nanog (B), Sox 2 (C) and Oct4 (D).

that overexpression of miR-145 induces tumorsphere differentiation and inhibits carcinogenesis and invasion of the tumorsphere. When we employed si-Ad-miR-145 to knockdown miR-145 in tumorspheres, we found increased levels of core TFs that were involved in the self-renewal, proliferation and invasion of tumorspheres (27). We also described an association between miR-145 levels and survival of CC patients based on data from TGCA. This comparison showed that patients with higher levels of miR-145 (and in combination with low Sox2, Nanog and Oct4 levels) had higher median survival than patients with lower miR-145 levels. From the TCGA database, it could be concluded miR-145 was an independent prognosis marker for CC patients. Taken together, these results suggest that miR-145 downregulates expression of core TFs to induce CT differentiation. Hence, we demonstrated that miR-145 is involved in cervical carcinogenesis.

Recently, unmodified miR-145 delivered by peptide-based vectors applied through a system or location in a mouse model of colon carcinoma resulted in a 40 or $60 \%$ decrease in tumor growth, with concomitant repression of ERK5 and c-Myc protein levels compared with negative controls, respectively (28). miR-145 can increase the sensitivity of tumors to chemotherapy or radiotherapy, and miR-145 treatment can increase sensitivity to 5-fluorouracil in gastric cancer cells (29). Similarly, local injection of Ad-miR-145 results in significant suppression of tumor growth in orthotopic mouse models of breast cancer (22). In our study, Ad-miR-145 effectively inhibited the growth and invasion of tumor cells by decreasing expression of core stem cell TFs in null-mice. These results suggest that miR-145 could be a potential target of therapy for cervical carcinoma.

\section{Acknowledgements}

This project was supported by the Natural Science Foundation of Shanxi Province, China (grant no. 2013JM4012) and the Key projects of Hubei Provincial Department of Education (grant no. 20162103), Natural Science Foundation of Hubei Province, China (grant no. 2016CFB409) .

\section{References}

1. Li J, Kang LN and Qiao YL: Review of the cervical cancer disease burden in mainland China. Asian Pac J Cancer Prev 12: 1149-1153, 2011. 
2. Siegel RL, Miller KD and Jemal A: Cancer statistics, 2015. CA Cancer J Clin 65: 5-29, 2015.

3. Shepherd JH: Cervical cancer. Best Pract Res Clin Obstet Gynaecol 26: 293-309, 2012.

4. Shi JF, Canfell K, Lew JB and Qiao YL: The burden of cervical cancer in China: Synthesis of the evidence. Int J Cancer 130 641-652, 2012

5. Baccelli I and Trumpp A: The evolving concept of cancer and metastasis stem cells. J Cell Biol 198: 281-293, 2012.

6. Liu A, Yu X and Liu S: Pluripotency transcription factors and cancer stem cells: Small genes make a big difference. Chin J Cancer 32: 483-487, 2013.

7. Li D, Mei H, Qi M, Yang D, Zhao X, Xiang X, Pu J, Huang K, Zheng L and Tong Q: FOXD3 is a novel tumor suppressor that affects growth, invasion, metastasis and angiogenesis of neuroblastoma. Oncotarget 4: 2021-2044, 2013.

8. Wang HY, Lian P and Zheng PS: SOX9, a potential tumor suppressor in cervical cancer, transactivates p21WAF1/CIP1 and suppresses cervical tumor growth. Oncotarget 6: 20711-20722, 2015.

9. Shen L, Huang X, Xie X, Su J, Yuan J and Chen X: High expression of SOX 2 and OCT4 indicates radiation resistance and an independent negative prognosis in cervical squamous cell carcinoma. J Histochem Cytochem 62: 499-509, 2014.

10. Ji J, Wei X and Wang Y: Embryonic stem cell markers Sox-2 and OCT4 expression and their correlation with WNT signal pathway in cervical squamous cell carcinoma. Int J Clin Exp Pathol 7: 2470-2476, 2014.

11. Ding Y, Yu AQ, Li CL, Fang J, Zeng Y and Li DS: TALENmediated Nanog disruption results in less invasiveness, more chemosensitivity and reversal of EMT in Hela cells. Oncotarget 5: 8393-8401, 2014

12. Mathieu J and Ruohola-Baker H: Regulation of stem cell populations by microRNAs. Adv Exp Med Biol 786: 329-351, 2013

13. Xu N, Papagiannakopoulos T, Pan G, Thomson JA and Kosik KS: MicroRNA-145 regulates OCT4, SOX2, and KLF4 and represses pluripotency in human embryonic stem cells. Cell 137: 647-658, 2009.

14. Khan S, Ebeling MC, Zaman MS, Sikander M, Yallapu MM, Chauhan N, Yacoubian AM, Behrman SW, Zafar N, Kumar D, et al: MicroRNA-145 targets MUC13 and suppresses growth and invasion of pancreatic cancer. Oncotarget 5: 7599-7609, 2014.

15. Cui SY, Wang R and Chen LB: MicroRNA-145: A potent tumour suppressor that regulates multiple cellular pathways. J Cell Mol Med 18: 1913-1926, 2014

16. Arndt GM, Dossey L, Cullen LM, Lai A, Druker R, Eisbacher M, Zhang C, Tran N, Fan H, Retzlaff K, et al: Characterization of global microRNA expression reveals oncogenic potential of miR-145 in metastatic colorectal cancer. BMC Cancer 9: 374 2009.
17. Inamoto $\mathrm{T}$, Taniguchi $\mathrm{K}$, Takahara K, Iwatsuki A, Takai T, Komura K, Yoshikawa Y, Uchimoto T, Saito K, Tanda N, et al: Intravesical administration of exogenous microRNA-145 as a therapy for mouse orthotopic human bladder cancer xenograft. Oncotarget 6: 21628-21635, 2015.

18. Wu Y, Liu S, Xin H, Jiang J, Younglai E, Sun S and Wang H: Up-regulation of microRNA-145 promotes differentiation by repressing OCT4 in human endometrial adenocarcinoma cells. Cancer 117: 3989-3998, 2011.

19. Zhou X, Gao Q, Wang J, Zhang X, Liu K and Duan Z: Linc-RNA-RoR acts as a 'sponge' against mediation of the differentiation of endometrial cancer stem cells by microRNA-145. Gynecol Oncol 133: 333-339, 2014

20. Alvarado AG, Turaga SM, Sathyan P, Mulkearns-Hubert EE, Otvos B, Silver DJ, Hale JS, Flavahan WA, Zinn PO, Sinyuk M, et al: Coordination of self-renewal in glioblastoma by integration of adhesion and microRNA signaling. Neuro-oncol 18: 656-666, 2016.

21. Wang Y, Xu Z, Jiang J, Xu C, Kang J, Xiao L, Wu M, Xiong J, Guo $X$ and Liu $\mathrm{H}$ : Endogenous miRNA sponge lincRNA-RoR regulates Oct4, Nanog, and Sox 2 in human embryonic stem cell self-renewal. Dev Cell 25: 69-80, 2013.

22. Kim SJ, Oh JS, Shin JY, Lee KD, Sung KW, Nam SJ and Chun KH: Development of microRNA-145 for therapeutic application in breast cancer. J Control Release 155: 427-434, 2011.

23. Crosbie EJ, Einstein MH, Franceschi S and Kitchener HC: Human papillomavirus and cervical cancer. Lancet 382: 889-899, 2013.

24. Gunasekharan V and Laimins LA: Human papillomaviruses modulate microRNA 145 expression to directly control genome amplification. J Virol 87: 6037-6043, 2013.

25. Luo W, Li S, Peng B, Ye Y, Deng X and Yao K: Embryonic stem cells markers SOX2, OCT4 and Nanog expression and their correlations with epithelial-mesenchymal transition in nasopharyngeal carcinoma. PLoS One 8: e56324, 2013.

26. Wang X, Tang S, Le SY, Lu R, Rader JS, Meyers C and Zheng ZM: Aberrant expression of oncogenic and tumor-suppressive microRNAs in cervical cancer is required for cancer cell growth. PLoS One 3: e2557, 2008.

27. Chhabra R and Saini N: MicroRNAs in cancer stem cells: Current status and future directions. Tumour Biol 35: 8395-8405, 2014.

28. Ibrahim AF, Weirauch U, Thomas M, Grünweller A, Hartmann RK and Aigner A: MicroRNA replacement therapy for miR-145 and miR-33a is efficacious in a model of colon carcinoma. Cancer Res 71: 5214-5224, 2011

29. Takagi T, Iio A, Nakagawa Y, Naoe T, Tanigawa N and Akao Y: Decreased expression of microRNA-143 and -145 in human gastric cancers. Oncology 77: 12-21, 2009. 